\title{
Publisher Correction: Lineage plasticity in cancer: a shared pathway of therapeutic resistance
}

Álvaro Quintanal-Villalonga, Joseph M. Chan, Helena A. Yu(D), Dana Pe'er, Charles L. Sawyers (1), Triparna Sen (1) and Charles M. Rudin (1)

Nature Reviews Clinical Oncology (2020) https://doi.org/10.1038/s41571-020-0340-z Published online 9 March 2020

In the originally published version of the Review from Quintanal-Villalonga et al., the name of the author Triparna Sen was not listed correctly and appeared in the wrong order (Sen Triparna). This has been corrected in the HTML and PDF versions of the manuscript.

https://doi.org/10.1038/s41571-020-0355-5 I Published online 17 March 2020

(c) Springer Nature Limited 2020 